Dr. Jorge Clemente Mediavilla

Universidad Complutense de Madrid

@ jclemen@ucm.es

(iD) $0000-0002-9819-1129$

Dra. Ana Visiers Elizaincín

Universidad Complutense de Madrid

@ avisiers@ucm.es

(iD) 0000-0003-1729-5645
Recibido / Received 20 de marzo de 2017

- Aceptado / Acepted 9 de abril de 2018

- Páginas / Pages De la 99 a la 108

- ISSN: 1885-365X

\title{
El rol del Producer en las agencias publicitarias de España
}

The role of the Producer in advertising agencies in Spain

La crisis económica de 2008 supuso la desaparición de los departamentos de producción en muchas agencias de publicidad en España. Con motivo de la reducción de la inversión publicitaria, las agencias decidieron ahorrar costes y se comenzó a contratar productores externos para el desarrollo de las producciones audiovisuales. A través de entrevistas en profundidad a anunciantes, agencias y productoras se ha analizado qué ha supuesto la pérdida de estos departamentos de producción, las desventajas de un productor que no forma parte de la agencia y la aparición de nuevas figuras en los anunciantes que realizan la labor del Producer de agencia.

PALABRAS CLAVE: productor, agencia, publicidad, crisis.

The economic crisis of 2008 meant the disappearance of the production departments in many advertising agencies in Spain. Due to the reduction of the investment in advertising, the agencies decided to save costs and began hiring external producers for the development of audiovisual productions. Through in-depth interviews with advertisers, agencies and producers, this paper analyzes what the loss of these production departments meant, the disadvantages of an external producer and the appearance of new figures in the advertisers who carry out the work of the agency Producer.

KEY WORDS: agency, producer, advertising, crisis.

\section{Introducción}

El proceso de producción audiovisual publicitario responde a un protocolo donde todos los que participan saben qué lugar ocupan y qué labores deben desempeñar para el correcto desarrollo de un spot, desde que el anunciante encarga a la agencia de publicidad una campaña audiovisual, hasta su emisión en televisión, cine o internet.

Debido a la crisis económica se comenzaron a apreciar unos cambios en esos procesos. La inversión de los anunciantes se vio reducida de manera significativa, lo que afectó a la remuneración de las agencias y directamente a los presupuestos destinados a las 
producciones audiovisuales. Asimismo, las agencias y productoras se vieron obligadas a disminuir sus gastos, lo que ha afectado a su estructura y a su forma de trabajar.

El objetivo principal de la presente investigación consiste en evaluar los departamentos de producción de las agencias de publicidad, con especial atención al rol del Producer. Se trata de averiguar de qué forma ha influido la crisis económica en la estructura de la agencia y en concreto en el departamento de producción audiovisual. Tras analizar cuáles son las principales funciones de un departamento de producción, se tratará de dar respuesta a cuestiones como la organización de las agencias ante un proceso de producción, y cómo influye la figura del Producer en el trabajo de los demás profesionales de las agencias, tanto creativos como departamentos de cuentas.

Por último, se examinará de qué forma perciben productoras y postproductoras el trabajo del departamento de producción de las agencias en la actualidad. Todo ello, sin olvidar de qué forma han podido afectar todos estos cambios al anunciante, y las ventajas y desventajas de las nuevas incorporaciones en sus procesos de producción. Un análisis desde el punto de vista de todos los que intervienen en este proceso podría confirmar de qué forma deben trabajar los departamentos de producción de las agencias de publicidad.

\section{Estado de la cuestión}

Para introducir la figura del director de producción y los productores audiovisuales publicitarios, se ha investigado sobre el comienzo de esta profesión. Pérez Ruiz (2003, p. 175) explica la estructura de las agencias y ya desde el inicio de la publicidad audiovisual aparece la figura del realizador dentro del departamento creativo de las agencias. Un departamento que contaba con un director creativo, redactores de textos, directores de arte y la sección de realización. El estudio estaba dirigido por un jefe de departamento del que dependían el resto de los profesionales y el director creativo era el que daba el visto bueno a su trabajo.

Ya en los años 70 y también dentro del departamento creativo aparece la figura del productor: «la producción se convirtió en la tercera función del departamento de creatividad» (Pérez Ruiz, 2003, p. 339). Entre otras tareas, el productor se encargaba de la búsqueda de modelos para los spots o de la relación con los estudios de grabación y sonorización, y siempre bajo la supervisión de los creativos.

Son numerosos los autores que recogen en sus investigaciones la integración de la producción en el departamento creativo. White (2010, p. 8) explica que el departamento que crea y produce los anuncios está compuesto por escritores, artistas y productores. Para Ortega Martínez (2004, p. 305), el departamento cuenta con un director creativo del que dependen los directores de arte y redactores de texto y los productores o realizadores.

Como se puede ver, las agencias de una u otra manera contaban con estos profesionales dentro de su estructura, aunque conviene aclarar, como apunta Fandiño Alonso (2003, p. 44), que en agencias pequeñas los encargados de la producción ante la ausencia de estos profesionales, eran los creativos.

Victoria Mas (2005, p. 116) explica quiénes componen el departamento de producción de una agencia. El director del departamento es el director de producción, que organiza y controla el trabajo de su equipo. A su vez, nombra dos responsables: uno de gráfica y otro 
de producción audiovisual. Cada uno de ellos contará con personal y técnicos más o menos especializados. $Y$ todos ellos reportarán al director creativo.

Carvajal Sáenz destaca la labor de representar a agencia y anunciante durante el proceso que realizan las empresas subcontratadas:

\begin{abstract}
El departamento de producción audiovisual suele estar dirigido por un Producer o responsable de la gestión de los proyectos, que es el conductor de los intereses publicitarios, técnicos, y económicos, de la agencia a quien representa y subsidiariamente de su cliente anunciante, durante el proceso de producción de cualquier acción audiovisual generado por aquella, frente a los proveedores concertados (2016, p. 20).
\end{abstract}

Para Victoria Mas, la gestión de buenos proveedores por parte del departamento de producción puede suponer un valor añadido muy importante para su agencia: «Tienen una función relevante de realización de presupuestos para la planificación previa de la campaña al cliente y presentación de la propuesta. Unos proveedores profesionales con unas tarifas adecuadas a su trabajo pueden suponer una importante ventaja competitiva para la agencia de publicidad» (2005, p. 117).

Baldwin (1989, p. 190) sostiene que el Producer tiene una labor complicada, ya que tiene que realizar una interpretación de la creatividad y al mismo tiempo, conocer los aspectos técnicos de la producción. Ahí radica la dificultad, en captar la intención de lo dibujado en el story board y ajustar la producción a esa intención, pero con un presupuesto marcado. Debe hacer balance entre las consideraciones artísticas y el coste.

Resulta relevante que su trabajo se desarrolle desde el momento en que los creativos empiezan a trabajar en una campaña. Carvajal Sáenz (2016, p. 20) lo explica así:

Debe estar presente desde el momento mismo de la concepción de la creatividad, participando activamente en cuantas aportaciones fueran necesarias al equipo creativo, para asistirle en su proceso de creación y ofrecerle información acerca de la viabilidad de sus ideas y de los métodos, tecnologías, profesionales o referencias que puedan servir de utilidad en el avance de sus creatividades.

Fandiño Alonso (2003, p. 44) es de la misma opinión, ya que afirma que las aclaraciones y especificaciones técnicas que realiza el departamento de producción pueden ayudar en gran medida a la toma de decisiones sobre la creatividad.

Para Book, Cary y Tannenbaum (1984, p. 193) tiene que tener un perfil creativo, que ayude con imaginación y profesionalidad a vender el mensaje. Debe emplear su sentido artístico con su capacidad para hacer negocios, porque sólo con la combinación de ambos puede realizarse un spot eficaz.

Ràfols y Colomer (2003, p. 62) sin embargo afirma que «no interviene directamente sobre la creación, pero sí que de su gestión puede depender en buena medida que el proyecto esté a la altura de las expectativas creadas".

Una vez aprobado el guion, las funciones del Producer serán las de «asegurarse de que se elegirá al director y a la productora que mejor se ajustan al guion. Para tomar esta decisión, debe visionar varias presentaciones y preparar diversas entrevistas preliminares 
con unos cuantos directores y productoras cinematográficas» (Burtenshaw, Mahon y Barfoot, 2007, p. 49).

El Producer en este punto debe contar con una buena agenda de profesionales, habrá analizado las bobinas de todos ellos y sabrá en qué está especializado cada uno. Asimismo, conocerá los presupuestos que suelen manejar, para no proponer un proveedor que presente un presupuesto que no puedan elegir por motivos económicos (Rivero Sánchez, 1992, p. 369).

Brierley (2005, p. 58) considera que es en este punto, cuando ya está aprobada la idea, cuando el Producer debe organizar la producción a través de una productora de publicidad o negociar con directores de cine para realizar el spot.

El papel de negociación de un presupuesto es una de las funciones más importantes de un Producer. Davies (2014) afirma que si el presupuesto de una productora está por encima de la cantidad marcada por el anunciante, se puede conseguir que la productora lo ajuste, pero sólo si esta negociación se realiza entre la productora y un «experto comprador de sus servicios».

Con todo lo visto, se puede decir que un Producerde agencia debe poseer unos conocimientos técnicos y unas habilidades que como dice Frank Scherma en una entrevista realizada por Parpis, debe tener «un poco de psiquiatra, de programador, creativo, productor, conciliador, niñera, y a la vez, tendrá que ser los ojos y oídos de la agencia de publicidad» (Parpis, 1999).

Para Cury (2013, p. 52) la naturaleza de este negocio requiere que sea alguien con «un fuerte, pero a veces frágil, ego".

En definitiva, y como también afirma Rivero la función del Producer no es sencilla:

Existen pocos con alto grado de preparación, pero son personas muy buscadas por las agencias de publicidad y bien pagadas, pues no hay que olvidar que gran parte del presupuesto global de una campaña de publicidad va destinado a producción, cada día más elevado y que muchos clientes llegan a dedicar el $10 \%$ del volumen total de la inversión de la campaña en medios (1992, p. 368).

\section{Metodología}

Para llevar a cabo la presente investigación se ha optado por una técnica de carácter cualitativo, como es la entrevista en profundidad a profesionales del sector. La muestra elegida abarca todas las fases del proceso de producción audiovisual: anunciantes, agencias de publicidad, productoras y postproductoras, que reflejan las distintas formas de producir que actualmente se están dando en el sector.

Según Ruiz Olabuénaga (2012, p. 55), el objetivo de la investigación cualitativa es conocer, más que describir unos hechos concretos, a través de las experiencias de los participantes, utilizando de forma fundamental el lenguaje y no los números o las estadísticas. El interés no está en llegar a resultados que permitan generalizar sino la captación de la realidad en su contexto recogida a través de diversas experiencias.

Las entrevistas se realizaron en el período comprendido entre octubre de 2016 y febrero de 2017 en Madrid. Todos los entrevistados son profesionales conocedores del proceso de producción publicitaria antes y después de la crisis económica, con la finalidad de comprender cómo ha afectado la crisis al sector de producción audiovisual publicitario. 
De acuerdo a la definición de Valles (2002, p. 20) que centra las entrevistas «en las experiencias subjetivas de la gente expuesta a la situación, con el propósito de contrastar las hipótesis y averiguar respuestas o efectos no anticipados", se plantean entrevistas focalizadas en los actores principales del proceso audiovisual. ¿Quiénes mejor que los protagonistas para hablar de este proceso?

Se realizaron 23 entrevistas en profundidad a profesionales dentro de cada una de las fases de producción publicitaria:

1. Anunciantes de marcas líderes en su sector y de productos muy distintos: alimentación, bebidas, telefonía y banca.

2. Cost controller y consultores del anunciante.

3. Directores de producción de agencia y Producers.

4. Directores de cuentas y directores creativos ejecutivos de agencias nacionales y multinacionales.

5. Productoras.

6. Postproductoras.

Se debe destacar que, autores como López Estrada y Deslauriers (2011, p. 9), recomiendan que las entrevistas se realicen asegurando el anonimato y recordando a los entrevistados el objetivo académico de las mismas, algo que se respetó escrupulosamente en nuestro caso.

Asimismo, no se conseguirían los mismos resultados si sólo dieran su opinión los Producers ya que se necesita conocer también el sentir de los anunciantes, consultores, creativos y cuentas, productoras y postproductoras. Sólo de esta forma se puede estudiar la realidad del Producer en su contexto. No obstante, en el caso de los Producers también se ha considerado relevante realizar una muestra de los distintos perfiles que existen hoy en día: directores de producción en agencia, Producers que trabajan siempre para una misma agencia pero no forman parte de la plantilla de la empresa, y Producers freelancer que realizan distintos proyectos y no siempre con la misma agencia de publicidad.

\section{Análisis}

En 1996 en Barcelona, y en el año 2000 en Madrid, surge la primera empresa de servicios de producción externos a la agencia de publicidad. En ese momento, los departamentos de producción audiovisual de las agencias eran fuertes, pero el volumen de producción era muy alto, de forma que ya se empiezan a subcontratar a Producers que forman parte de la plantilla de Central de Producers y dan servicio a varias agencias de publicidad, normalmente bajo la tutela de los directores de producción de las agencias para las que trabajan.

Pero no es hasta 2011 cuando esta misma empresa desarrolla una campaña, protagonizada por varios directores creativos y directores de producción, con el propósito de demostrar la importancia del trabajo de un buen Producers: "Hemos detectado que hay agencias que se han subido al carro del "yo me lo guiso, yo me lo como", arrancando las producciones de las diferentes disciplinas, ya sea un spot, una foto, un evento, etcétera, de forma poco ortodoxa, provocando verdaderos quebraderos de cabeza entre los equipos de cuentas y los 
creativos», comentaban a la revista Anuncios (El valor de un buen producer, 2011).

Carvajal Sáenz hace mención también a esta desaparición del departamento de producción audiovisual en las agencias de publicidad:

Las diferentes reestructuraciones efectuadas buscando la optimización de servicios, obligaron a algunas agencias a prescindir de este departamento, y se empezaron a encargar los servicios de supervisión y control de la producción a proveedores externos, eliminando así el gasto fijo que ello pudiera suponer en su presupuesto general (2016, p. 20).

Lo que parece indiscutible hoy es que el departamento de producción se reduce ya a unas pocas agencias, ya que en la mayoría de ellas han prescindido de esta figura, en gran medida sustituida por agentes externos. Los entrevistados apuntaron que las causas de esta desaparición o externalización se han debido a la crisis económica y a los recortes que, en consecuencia, han sufrido las agencias.

Son las propias agencias las que reconocieron en las entrevistas realizadas que los motivos de esta desaparición o externalización del departamento de producción se debió a razones puramente financieras. En el momento en que los anunciantes reducen su inversión, las agencias tienen que reducir sus costes fijos, y ante estructuras cada vez más raquíticas, la única forma de tener un departamento de producción solvente fue externalizarlo. Los Producers entrevistados, sin embargo, van un poco más allá y explican que los anunciantes eliminaron el fee que pagaban a las agencias por las producciones, de forma que su implicación en los procesos de producción audiovisual se volvía insostenible.

$Y$ aunque los anunciantes aseguraron que se estaban acostumbrando a que las agencias hubieran prescindido de los departamentos de producción dentro de sus estructuras con motivo de la crisis económica, también confirmaron que les gustaría volver a contar con ellos ya que, también bajo su punto de vista, los presupuestos han comenzado a mejorar. Para los anunciantes la figura del Producer es una garantía en el proceso de producción. Ponen en valor los conocimientos de estos profesionales, su relación con el departamento creativo y su papel como coordinador e integrador dentro de la producción. Algunos de ellos entienden que si el volumen de producción audiovisual no es elevado, es posible tener esa figura fuera de la agencia, pero no lo conciben en el caso de agencias con anunciantes que tienen gran volumen de producción anual.

Los Cost controllery consultores entrevistados insistieron en la importancia de la cualificación del Producery su papel crucial en el proceso de producción. Entre otras cualidades destacan su labor como mano derecha del director creativo, que conoce la idea desde su misma concepción, y que interviene dando forma a esas propuestas, ya que, con su conocimiento del mercado, puede dar referencias y aportar soluciones a todas aquellas dudas que se planteen desde el comienzo del proceso audiovisual, asegurando de este modo que la producción se realizará cumpliendo con el presupuesto y el timing marcado por el anunciante.

Los propios Producers destacaron también que lo mismo que no se concibe la externalización de un departamento creativo, tampoco debería ser una opción para el departamento de producción, que en su opinión debe formar parte de la agencia. Entienden que su trabajo conlleva tantas horas de dedicación que resulta imposible que una agencia 
lo asuma si el anunciante no quiere pagarlo. También ellos destacan su papel desde la creación, la búsqueda de productoras, la supervisión de presupuestos y tratamientos de los realizadores para llegar a la mejor opción, el control de la preproducción, el rodaje y la postproducción, hasta la entrega de las copias de emisión. Papel que no puede realizar un perfil "junior" porque entre otras cosas, solo con la experiencia un Producer puede argumentar un presupuesto, aportar soluciones a distintos imprevistos, o ahorrar costes a un anunciante con la gestión de los derechos de un spot. Cuando un Producer hace bien su trabajo se cumplen los procesos, se realiza una elección acertada de la productora, se le explica bien la creatividad de forma que pueda presupuestar correctamente y el anunciante entienda esos gastos, los timings estén ajustados a la producción, etc. En opinión de algunos de ellos, cuando su trabajo está bien hecho, es fácil pasar inadvertidos.

No obstante, los directores creativos y directores de cuentas resaltaron en las entrevistas el valor del Producer. De hecho, todos ellos comenzaron en agencias con departamentos de producción sólidos, y consideraban al director de producción como su mano derecha a la hora de diseñar una campaña, y sólo tras estudiar juntos las posibilidades de una campaña, se la presentaban al anunciante. Además, los creativos consideraron que la labor del Producer no es fácil, porque no sólo deben ganarse la confianza del anunciante, sino que son los responsables de explicar y enfrentarse al resto de la agencia cuando la opción a presentar es inviable desde el punto de vista de la producción.

Los directores de cuentas consideraron también haber salido perjudicados con esta reestructuración de las agencias. En muchos casos tuvieron que asumir algunas de las funciones, con el problema de no tener criterio para entender si con ese presupuesto o ese timing impuesto por el cliente serían capaces de sacar adelante una campaña de calidad.

Y en ese punto, también las productoras confirmaron que, al no tener un filtro en las agencias, en numerosas ocasiones recibían guiones de los departamentos creativos imposibles de producir. Productoras y postproductoras se lamentaron de la posición actual de los Producers. Destacaron que perfiles "junior" realizaban labores de simples transmisores de información, sin criterio para dar una recomendación o mostrar su inquietud ante determinadas propuestas.

Cabe destacar que la posición de todos los entrevistados sobre los Producers externos a la agencia fue unánime. Desde el punto de vista del anunciante no sentían que les conociera, que entendiera sus intereses de la misma forma que un Producer que forma parte de su agencia. Los propios Producers confirmaron también que cuando actúan como externos tienen la principal dificultad de comenzar con proyectos en los que la creatividad ya ha sido aprobada. Asimismo, destacaron que se encontraban en una posición complicada al tener que ganarse no sólo la confianza de los anunciantes, sino también la de creativos y cuentas. Los directores creativos afirmaron que para ellos es difícil, ya que esa persona entraba para un proyecto, pero no estaba en el día a día de la agencia, y por ello consideran que la comunicación no es igual porque no es parte del equipo.

No obstante, conviene aclarar que todavía algunas agencias españolas mantienen sus departamentos de producción. Uno de los cuatro Producers entrevistados forma parte de la plantilla de la agencia, y aseguró que fueron los creativos los que consiguieron que se mantuviera al menos al director de producción, aunque se tuvieran que subcontratar refuerzos para algunas producciones.

Del total de los 23 profesionales entrevistados en esta investigación, tan solo tres 
consideraron que se pudiera prescindir del Producer de agencia, y que su papel lo pudiera desempeñar el Anunciante internalizando sus funciones. Sin embargo, cabe resaltar que los tres llegaron a esa conclusión en el momento en que el departamento de producción deja de ser parte de las agencias de publicidad, y pasa a ser, en la mayoría de los casos, una figura externa. Se trata de un Anunciante que comentó haber introducido en su estructura un departamento de producción que trabaja directamente con los departamentos de Cuentas y Creatividad de las distintas agencias; y una Productora y una Postproductora que consideraron que el papel que realiza en la actualidad un Producer externo podría ser sustituido por un Producer que trabaje en exclusividad para un Anunciante.

\section{Conclusiones}

Parece claro que los departamentos de producción de las agencias han ido desapareciendo. El motivo fundamental ha sido la crisis económica, que ha llevado a una reducción de las producciones y los presupuestos y, por tanto, las agencias toman la decisión de externalizar estas funciones para ahorrar unos costes fijos. Sin embargo, para todos los entrevistados, las funciones que ejercían los Producers audiovisuales eran de enorme importancia en el proceso. Por ese motivo, la mayoría piensa que debe ser un departamento que vuelva al seno de las agencias y con el mismo rango que los directores creativos. En ese sentido y siguiendo los resultados obtenidos, las agencias deben contar por lo menos con un director de producción que se haga cargo de todas las producciones audiovisuales, aunque pueda contar además con profesionales "freelancer" en aquellos momentos en que las producciones solaparan su proceso de elaboración, con el fin de no encarecer de nuevo las estructuras de las agencias si el volumen no es igual todos los meses del año.

No obstante, se considera que contar con un profesional de la producción en la propia estructura del anunciante, que vele por la mejor calidad al mejor precio, es beneficioso para la producción. La figura del Producer del anunciante no debe estar reñida con la del Producer de agencia, sino todo lo contrario. Teniendo en cuenta la enorme inversión en medios que realiza un anunciante puede resultar conveniente contar con las dos figuras durante el proceso: un Producer de anunciante facilitaría la producción al contar con un interlocutor en el cliente que entendiera todo aquello que le plantee de forma transparente un Producer de agencia. Y éste al mismo tiempo podría trabajar de la mano del creativo aportando todos sus conocimientos para conseguir una mayor calidad con el presupuesto adjudicado a cada producción.

Otra cuestión que conviene resaltar es la necesidad de procurar la correcta formación de estos profesionales, considerando que en muchos casos han sido relegados de sus funciones y ya no pueden enseñar a los jóvenes la realidad de una profesión que por unos motivos u otros en los últimos años ha sido vapuleada y menospreciada. 


\section{Bibliografía}

BALDWIN, Huntley (1989). How to create effective TV commercials. 2nd. ed. ed. Lincolnwood, Illinois: NTC Business Books.

BOOK, Albert C., CARY, Norman D. y TANNENBAUM., Stanley I. (1984). The radio and television commercial. 2nd ed. Chicago: Crain Books.

BRIERLEY, Sean (2005). The advertising handbook. Routledge.

BURTENSHAW, Ken, MAHON, Nik y BARFOOT, Caroline (2007). Principios de publicidad: El proceso creativo: agencias, campañas, medios, ideas y dirección de arte. Barcelona: Gustavo Gili.

CARVAJAL SÁENZ, Alejandro (2016). El proceso de producción audiovisual. Punto Rojo Libros.

CURY, Ivan (2013). TV commercials: How to make them. 1st ed. Focal Press.

DAVIES, Steve (12 de junio de 2014). Cannes perspectives: No shortcut to quality. Campaign. Recuperado de http://www.campaignlive.co.uk/article/cannes-perspectives-no-shortcut-quality/1297073

El valor de un buen producer (11 de febrero de 2011). Anuncios. Recuperado de http://www.anuncios. com/campana-creatividad/mas-anuncios/1054529008001/valor-buen-producer.1.html

LÓPEZ ESTRADA, Raúl Eduardo y DESLAURIERS, Jean-Pierre (2011). La entrevista cualitativa como técnica para la investigación en trabajo social. Margen: revista de trabajo social y ciencias sociales, 61. Recuperado de: http://www.margen.org/suscri/margen61/lopez.pdf

FANDIÑO ALONSO, Jaime (2003). Introducción á producción publicitaria en medios audiovisuais. Vigo: Universidade de Vigo.

ORTEGA MARTINEZ, Enrique (2004). La comunicación publicitaria. $2^{\mathrm{a}}$ ed. Madrid: Pirámide.

PARPIS, Eleftheria (1999). Focus: Agency producer. Adweek.

PÉREZ RUIZ, Miguel Ángel (2003). La transición de la publicidad española: anunciantes, agencias, centrales y medios, 1950-1980. Madrid: Fragua.

RÀFOLS, Rafael; COLOMER, Antoni (2003). El diseño audiovisual. Barcelona: Editorial Gustavo Gili.

RIVERO SÁNCHEZ, José A. (1992). La productora de cine publicitario. Estructura técnica, organizativa y empresarial en la industria de la producción española. Micrográfica Publicacions de la Universitat Autònoma de Barcelona.

RUIZ OLABUÉNAGA, José Ignacio (2012). Metodología de la investigación cualitativa. 5a ed. Bilbao: Universidad de Deusto. 
VALLES, Miguel S. (2002). Entrevistas cualitativas. 32 Vol. Madrid: Centro de Investigaciones Sociológicas, 2002.

VICTORIA MAS, Juan Salvador (2005). Reestructuras del sistema publicitario. Barcelona: Ariel.

WHITE, Ed (2010). Agency producer Q\&A. Boards. 